\title{
General Linearized Theory of Quantum Fluctuations around Arbitrary Limit Cycles
}

\author{
Carlos Navarrete-Benlloch, ${ }^{1,2}$ Talitha Weiss, ${ }^{1,2}$ Stefan Walter, ${ }^{1,2}$ and Germán J. de Valcárcel ${ }^{3}$ \\ ${ }^{1}$ Max-Planck-Institut für die Physik des Lichts, Staudtstrasse 2, 91058 Erlangen, Germany \\ ${ }^{2}$ Institute for Theoretical Physics, Erlangen-Nürnberg Universität, Staudtstrasse 7, 91058 Erlangen, Germany \\ ${ }^{3}$ Departament d'Òptica, Facultat de Física, Universitat de València, Dr. Moliner 50, 46100 Burjassot, Spain
}

(Received 12 July 2017; published 26 September 2017)

\begin{abstract}
The theory of Gaussian quantum fluctuations around classical steady states in nonlinear quantum-optical systems (also known as standard linearization) is a cornerstone for the analysis of such systems. Its simplicity, together with its accuracy far from critical points or situations where the nonlinearity reaches the strong coupling regime, has turned it into a widespread technique, being the first method of choice in most works on the subject. However, such a technique finds strong practical and conceptual complications when one tries to apply it to situations in which the classical long-time solution is time dependent, a most prominent example being spontaneous limit-cycle formation. Here, we introduce a linearization scheme adapted to such situations, using the driven Van der Pol oscillator as a test bed for the method, which allows us to compare it with full numerical simulations. On a conceptual level, the scheme relies on the connection between the emergence of limit cycles and the spontaneous breaking of the symmetry under temporal translations. On the practical side, the method keeps the simplicity and linear scaling with the size of the problem (number of modes) characteristic of standard linearization, making it applicable to large (many-body) systems.
\end{abstract}

DOI: 10.1103/PhysRevLett.119.133601

Introduction.-The advent of modern quantum technologies has triggered the discovery of a plethora of optical, atomic, and solid state devices working in the quantum regime [1] (see also the starting paragraph of Ref. [2] and the references therein). A first-principles approach leads to a description of such devices as open quantum systems evolving according to nonlinear Hamiltonians and incoherent processes like dissipation [3-6]. Mathematically, one has to face master equations for the state of the system or quantum Langevin equations for its operators, which are in general impossible to solve exactly.

On the other hand, quantum nonlinearities are very difficult to observe in the laboratory and therefore most experiments are well described by effective linear models. The most widespread method for obtaining such linear models starting from nonlinear ones is the so-called standard linearization $[7,8]$. This consists of a Gaussianstate ansatz centered at the solution of the system's nonlinear equations in the classical limit [9]. The method combines incredible simplicity with pretty good accuracy, but can only be applied to stable solutions that are either stationary or follow trivially an external temporal modulation. This excludes the important case of systems that undergo self-oscillations. These are a particular case of a more general class of systems that being invariant under continuous transformations of some kind (e.g., time translations in the aforementioned case) develop a solution that breaks that invariance via spontaneous symmetry breaking. This is because Goldstone's theorem implies the existence of a zero eigenvalue of the linear stability matrix, and hence a direction of phase space that is not damped [10-14].
Standard linearization has been generalized to deal with the spontaneous symmetry breaking of spatial, polarization, and phase symmetries [10-19]. The method relies on phase-space representations of the state to keep track of the phase-space variable associated with the system's invariance, which will carry the largest part of the fluctuations. Then, the theory can be linearized with respect to any other phase-space variable.

However, an extension capable of dealing with selfpulsing solutions is still missing, because in this case symmetry breaking occurs in the very same variable that parametrizes the dynamics of the system: time. While this complicates enormously the problem compared to the other type of symmetries, here we succeed in generalizing standard linearization to one of the most prominent cases of self-pulsing solutions: those that are periodic in time, describing then a closed curve in phase space known as a periodic orbit or limit cycle [20-23]. These are ubiquitous to many optical phenomena, e.g., lasing [24-27], secondharmonic generation [7,28,29], and optomechanics [30-37], among others $[38,39]$. We show that the method approximates the quantum state of the system by a mixture of Gaussian states localized around the closed phase-space trajectory, and we give physical meaning to the way in which quantum fluctuations are distributed along these Gaussian states.

For convenience, we introduce the method for singlemode problems, using the driven quantum Van der Pol (VdP) oscillator [40-42] as an example. The simplicity of this model will allow for comparisons with full numerical simulations. The generalization to multimode problems is 
straightforward, and will be explored in the future for more practical and complex problems such as optomechanical cavities deep into the parametric instability regime $[43,44]$. Moreover, the complexity of the method scales only linearly with the number of modes, providing then an efficient route towards the analysis of many-body systems out of equilibrium such as optomechanical arrays [45-49] in the selfoscillating regime.

Van der Pol model.-The quantum model for a driven $\mathrm{VdP}$ oscillator consists of a single bosonic mode with annihilation operator $\hat{a}$, whose state $\hat{\rho}$ evolves according to the master equation $[40,41]$

$$
\frac{d \hat{\rho}}{d \tau}=\left[\frac{F}{\sqrt{\gamma}}\left(\hat{a}^{\dagger}-\hat{a}\right)+i \Delta \hat{a}^{\dagger} \hat{a}, \hat{\rho}\right]+\frac{\gamma}{2} \mathcal{D}_{a^{2}}[\hat{\rho}]+\mathcal{D}_{a^{\dagger}}[\hat{\rho}],
$$

where $\mathcal{D}_{J}[\hat{\rho}]=2 \hat{J} \hat{\rho} \hat{J}^{\dagger}-\hat{J}^{\dagger} \hat{J} \hat{\rho}-\hat{\rho} \hat{J}^{\dagger} \hat{J}$ and the bosonic operators satisfy canonical commutation relations $\left[\hat{a}, \hat{a}^{\dagger}\right]=1$. The Hamiltonian includes a coherent drive at rate $F / \sqrt{\gamma}>0$ detuned by $\Delta$ with respect to the natural oscillation frequency of the oscillator (note that we work in a picture rotating at the driving frequency). The model contains two incoherent terms as well, the first one corresponding to pairs of excitations lost irreversibly at rate $\gamma$ (nonlinear losses), and the second one to linear pumping. The rate of the latter is used to normalize the rest of the rates and frequencies, while its inverse normalizes time, so that $\tau, \gamma, F$, and $\Delta$ are dimensionless. We show later that with these choices the classical phase diagram of the system is determined uniquely by $F$ and $\Delta$, while $\gamma$ determines the strength of the quantum fluctuations.

The method is best introduced by mapping the master equation to a set of stochastic equations. This can be done with the help of phase-space quasiprobability distributions $[3,4,6,50]$ such as standard Wigner, Husimi, or GlauberSudharsan representations. Here, we choose the positive $P$ representation $[3,4,51,52]$ because, unlike the previous representations, it always leads to stochastic equations equivalent to the master equation without any approximation. This representation associates two independent stochastic variables that we denote by $\beta / \sqrt{\gamma}$ and $\beta^{+} / \sqrt{\gamma}$ with the annihilation and creation operators $\hat{a}$ and $\hat{a}^{\dagger}$, respectively, in such a way that normally ordered quantum expectation values and stochastic averages are related by $\left\langle\hat{a}^{\dagger m} \hat{a}^{n}\right\rangle=\left\langle\beta^{+m} \beta^{n}\right\rangle / \gamma^{(m+n) / 2}$ with $m, n \in \mathbb{N}$. Using standard techniques [3,4,51-53], we show in Ref. [54] that the stochastic amplitudes evolve according to

$\dot{\beta}=F+\left(1+i \Delta-\beta^{+} \beta\right) \beta+\sqrt{\gamma}[\sqrt{2} \xi(\tau)+i \beta \eta(\tau)]$,

$\dot{\beta}^{+}=F+\left(1-i \Delta-\beta^{+} \beta\right) \beta^{+}+\sqrt{\gamma}\left[\sqrt{2} \xi^{*}(\tau)-i \beta^{+} \eta^{+}(\tau)\right]$,

where $\eta(\tau), \eta^{+}(\tau)$, and $\xi(\tau)$ are independent white Gaussian noises (real the first two, and complex the last one) [54].
Limit cycles in the classical limit.-Coming from a normally ordered representation (where vacuum noise is already taken into account in the ordering), the equations above predict a large-amplitude coherent state for $\gamma \rightarrow 0$. We talk then about the classical limit. The remaining deterministic equation $\dot{\beta}=F+\left(1+i \Delta-|\beta|^{2}\right) \beta$ is a paradigm for synchronization phenomena [41], and its phase diagram is well known (we provide an overview of it in Ref. [54]). In general terms, its stationary solutions, corresponding to solutions oscillating at the driving frequency, are stable only provided a strong enough drive is fed; otherwise, the oscillations are not synchronized to the drive, so that for long times the system ends up in a nontrivial stable periodic solution $\bar{\beta}(\tau)=\bar{\beta}(\tau+T)$. In Fig. 1 we show an example of such limit cycle motion, where it can be appreciated that it describes a closed curve in phase space [Fig. 1(a)], with

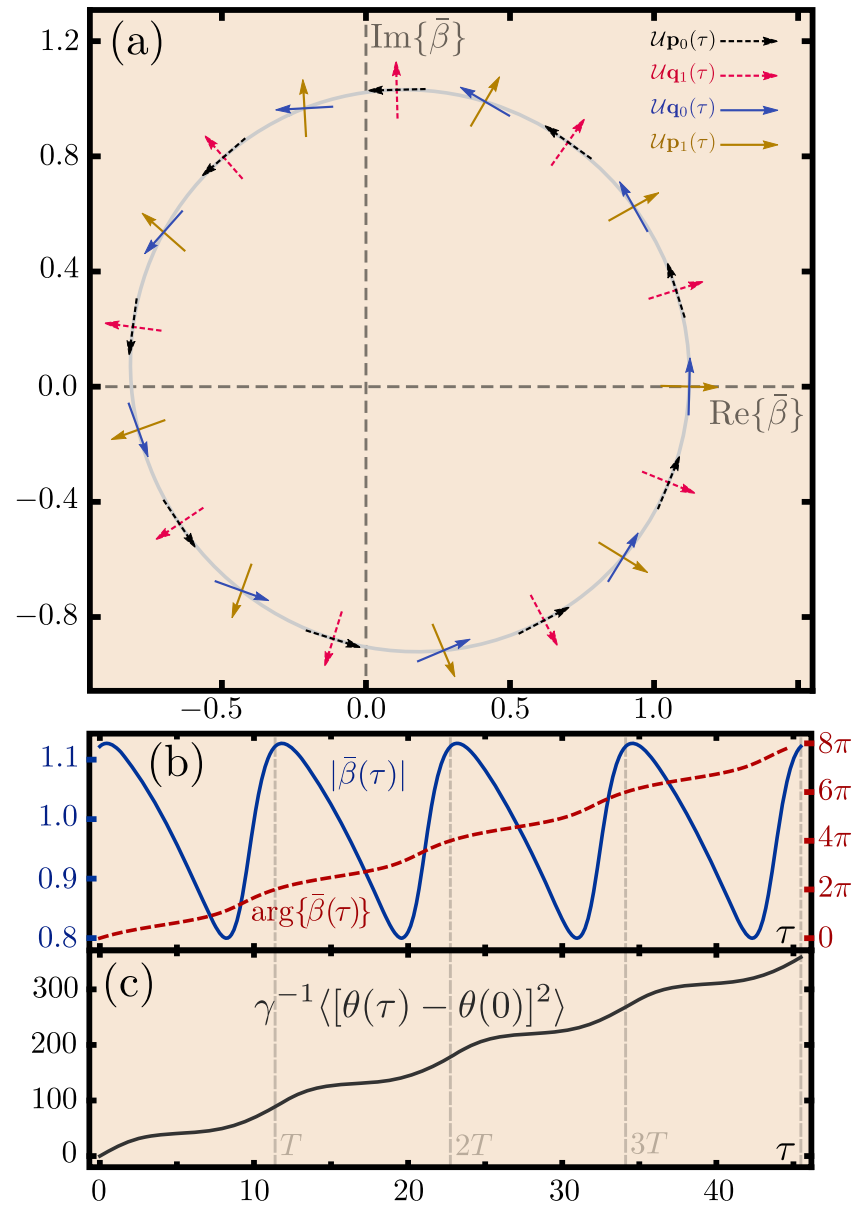

FIG. 1. Limit cycle emerging for $\Delta=\sqrt{0.4}$ and $F=\sqrt{0.1}$. (a) We show in gray the closed trajectory described in phase space. The arrows refer to the direction of the Floquet eigenvectors in selected points of the cycle. (b) Time evolution of the cycle's absolute value and phase. (c) Evolution of the variance of $\theta$, see Eqs. (3) and (8). Note that $\gamma$, which sets how relevant quantum fluctuations are, appears just as an absolute scale for the variance, whose dependence on time is set by the limit cycle's shape. 
an absolute value and a phase that oscillate periodically [Fig. 1(b)]. Note that analytical solutions for these limit cycles exist only in limited cases, and therefore one needs to find them numerically in general.

Linearization around limit cycles.-We are now able to introduce the linearization technique for quantum fluctuations around limit cycles. We start by expanding the stochastic amplitudes as

$$
\begin{aligned}
\beta(\tau+\theta) & =\bar{\beta}(\tau+\theta)+b(\tau+\theta), \\
\beta^{+}(\tau+\theta) & =\bar{\beta}^{*}(\tau+\theta)+b^{+}(\tau+\theta) .
\end{aligned}
$$

Here, $\theta$ determines at which point of the cycle the solution $\bar{\beta}(\tau+\theta)$ starts for $\tau=0$, and it is precisely the parameter that is not fixed by the classical equations of motion: $\bar{\beta}(\tau+\theta)$ is a solution of the equations for any choice of $\theta$. Owed to this symmetry, quantum fluctuations cannot be considered small in arbitrary points and directions of phase space, as nothing prevents them from acting on $\theta$ without resistance. Hence, in order for any linearized theory of quantum fluctuations to work, $\theta$ has to be taken as a variable itself (making it time dependent in the expansion above) and only then can the fluctuations $b$ and $b^{+}$be taken as small quantities. In addition, $\dot{\theta}$ can be taken as a small quantity as well, since variations of $\theta$ are induced by quantum noise, which is weak in the region of interest. Introducing Eq. (3) in Eq. (2), to first order in the small variables (including noise) we then get [54]

$$
\dot{\mathbf{b}}(\tau)+\mathbf{p}_{0}(\tau) \dot{\theta}(\tau)=\mathcal{L}(\tau) \mathbf{b}(\tau)+\sqrt{\gamma} \mathbf{n}(\tau),
$$

where $\mathbf{b}=\left(b, b^{+}\right)^{T}, \mathbf{p}_{0}=\left(\partial_{\tau} \bar{\beta}, \partial_{\tau} \bar{\beta}^{*}\right)^{T}, \mathbf{n}(\tau)=[\sqrt{2} \xi(\tau)+$ $\left.i \bar{\beta}(\tau) \eta(\tau), \sqrt{2} \xi^{*}(\tau)-i \bar{\beta}^{*}(\tau) \eta^{+}(\tau)\right]^{T}$, with the $T$ superindex denoting "transpose" (not to confuse with the time period), and

$$
\mathcal{L}(\tau)=\left(\begin{array}{cc}
1-2|\bar{\beta}(\tau)|^{2}+i \Delta & -\bar{\beta}(\tau)^{2} \\
-\bar{\beta}^{* 2}(\tau) & 1-2|\bar{\beta}(\tau)|^{2}-i \Delta
\end{array}\right)
$$

is the linear stability matrix. Note that the noise correlations can be written in the compact form $\left\langle n_{j}(\tau) n_{l}\left(\tau^{\prime}\right)\right\rangle=$ $N_{j l}(\tau) \delta\left(\tau-\tau^{\prime}\right)$, where $N_{j l}$ are the elements of the diffusion matrix

$$
\mathcal{N}(\tau)=\left(\begin{array}{cc}
-\bar{\beta}^{2}(\tau) & 2 \\
2 & -\bar{\beta}^{* 2}(\tau)
\end{array}\right) .
$$

As we will see, the introduction of $\theta(\tau)$ as an explicit variable will allow us to describe properly spontaneous temporal symmetry breaking and its associated undamped phase-space direction.

Floquet method and eigenvectors. - The main difference of Eq. (4) with respect to the linearized Langevin equations found in previous linearization methods is the time periodicity of $\mathbf{p}_{0}(\tau)$ and $\mathcal{L}(\tau)$. We deal with this by applying Floquet theory $[22,55]$ as we explain next.
Let us define the fundamental matrix $\mathcal{R}(\tau)$, which satisfies the initial value problem $\dot{\mathcal{R}}(\tau)=\mathcal{L}(\tau) \mathcal{R}(\tau)$ with $\mathcal{R}(0)=\mathcal{I}$, the latter being the identity matrix. From it, we further define the matrix $\mathcal{M}$ through $\exp (\mathcal{M} T)=\mathcal{R}(T)$, and the $T$-periodic matrix $\mathcal{P}(\tau)=\mathcal{R}(\tau) \exp (-\mathcal{M} \tau)$. Given the eigensystem $\left\{\mathbf{v}_{j}, \mathbf{w}_{j} ; \mu_{j}\right\}_{j=0,1}$ of $\mathcal{M}$, composed of right and left orthogonal $\left(\mathbf{w}_{j}^{\dagger} \mathbf{v}_{l}=\delta_{j l}\right)$ eigenvectors satisfying $\mathcal{M} \mathbf{v}_{j}=\mu_{j} \mathbf{v}_{j}$ and $\mathbf{w}_{j}^{\dagger} \mathcal{M}=\mu_{j} \mathbf{w}_{j}^{\dagger}$, we introduce the Floquet eigenvectors $\mathbf{p}_{j}(\tau)=\mathcal{P}(\tau) \mathbf{v}_{j}$ and $\mathbf{q}_{j}^{\dagger}(\tau)=\mathbf{w}_{j}^{\dagger} \mathcal{P}^{-1}(\tau)$. As we show along the next sections, knowledge of these vectors is enough to derive the linearized quantum properties of the system. To this aim, it is also convenient to point out that they satisfy the initial value problems

$$
\begin{array}{rlrl}
\dot{\mathbf{p}}_{j}(\tau) & =\left[\mathcal{L}(\tau)-\mu_{j}\right] \mathbf{p}_{j}(\tau), & \mathbf{p}_{j}(0)=\mathbf{v}_{j}, \\
\dot{\mathbf{q}}_{j}^{\dagger}(\tau)=\mathbf{q}_{j}^{\dagger}(\tau)\left[\mu_{j}-\mathcal{L}(\tau)\right], & \mathbf{q}_{j}^{\dagger}(0)=\mathbf{w}_{j}^{\dagger},
\end{array}
$$

and the orthogonality conditions $\mathbf{q}_{j}^{\dagger}(\tau) \mathbf{p}_{l}(\tau)=\delta_{j l} \forall \tau$, as is easily proven from their definition.

Let us now comment on the general properties of this eigensystem, which we prove in detail in Ref. [54]. There always exists a null eigenvalue, say $\mu_{0}=0$, with related (right) Floquet eigenvector $\mathbf{p}_{0}(\tau)$. This property is a byproduct of the spontaneous temporal symmetry breaking generated by the limit cycle (Goldstone theorem). In the single-mode case, there is just another eigenvalue, which is given by $\mu_{1}=\int_{0}^{T} d \tau \operatorname{tr}\{\mathcal{L}(\tau)\} / T$, and has associated (left) Floquet eigenvector $\mathbf{q}_{1}(\tau)=\left(-i \partial_{\tau} \bar{\beta}, i \partial_{\tau} \bar{\beta}^{*}\right)^{T} \times$ $\exp \left\{\int_{0}^{\tau} d \tau^{\prime} \operatorname{tr}\left\{\mathcal{L}\left(\tau^{\prime}\right)\right\}-\mu_{1} \tau\right\}$. This vector is the temporal counterpart of the linear or angular momentum found in previous works that deal with spatial symmetries [14].

Note that $\mathbf{p}_{0}(\tau)$ and $\mathbf{q}_{1}(\tau)$ are, respectively, the tangent and normal vectors of the limit cycle's trajectory, see Fig. 1(a). We haven't found explicit expressions of the other Floquet eigenvectors in terms of $\bar{\beta}(\tau)$, but they can always be found numerically in an efficient fashion, as we do for Fig. 1(a).

Diffusion of the temporal pattern.-As a first physical consequence of the properties above, we now show that $\theta$ is diffusing due to quantum noise, and hence quantum fluctuations smear off the classical periodic orbit.

In order to show this, we just need to apply $\mathbf{q}_{0}^{\dagger}(\tau)$ on Eq. (4), obtaining $d / d \tau\left(\mathbf{q}_{0}^{\dagger} \mathbf{b}+\theta\right)=\sqrt{\gamma} \mathbf{q}_{0}^{\dagger}(\tau) \mathbf{n}(\tau)$. Note that by taking $\theta$ as a variable in Eq. (3) we introduced a redundancy in the number of variables, which is now consistently removed by setting $\mathbf{q}_{0}^{\dagger} \mathbf{b}=0$ (in other words, introducing $\theta$ simply allowed us to track and give physical meaning to this part of the quantum fluctuations). The previous equation turns then into a diffusion equation for $\theta$, leading to a variance

$$
\left\langle[\theta(\tau)-\theta(0)]^{2}\right\rangle=\gamma \int_{0}^{\tau} d \tau^{\prime} \mathbf{q}_{0}^{\dagger}\left(\tau^{\prime}\right) \mathcal{N}\left(\tau^{\prime}\right) \mathbf{q}_{0}^{*}\left(\tau^{\prime}\right) .
$$


Note that the kernel is periodic, and therefore, the coarsegrained dynamics of $\theta$ corresponds to a diffusion process, with a variance increasing linearly with time, making $\theta$ fully undetermined asymptotically as shown in Fig. 1(c).

Steady state as a mixture of Gaussians. - The above considerations imply that the steady state is formed by a balanced mixture of Gaussian states, one for each value of $\theta$. As we prove below, the Wigner functions of these Gaussian states [56] are given by

$$
W(\mathbf{r}, \tau+\theta)=\frac{e^{-\frac{1}{2}[\mathbf{r}-\overline{\mathbf{d}}(\tau+\theta)]^{T} \bar{V}^{-1}(\tau+\theta)[\mathbf{r}-\overline{\mathbf{d}}(\tau+\theta)]}}{2 \pi \sqrt{\operatorname{det}\{\bar{V}(\tau+\theta)\}}},
$$

where $\mathbf{r}=(x, p)^{T}$ is the coordinate vector in phase space, and the mean vector and covariance matrix are given by

$$
\begin{aligned}
\overline{\mathbf{d}}(\tau) & =\mathcal{U}\left[\bar{\beta}(\tau), \bar{\beta}^{*}(\tau)\right]^{T} / \sqrt{\gamma}, \\
\bar{V}(\tau) & =\mathcal{I}+C(\tau) \mathcal{U} \mathbf{p}_{1}(\tau) \mathbf{p}_{1}^{T}(\tau) \mathcal{U}^{T} .
\end{aligned}
$$

$\mathcal{U}=\left(\begin{array}{cc}1 & 1 \\ -i & i\end{array}\right)$ is the matrix that connects the complex representation of the bosonic mode to its real representation in phase space, and

$$
C(\tau)=\lim _{\tau \rightarrow \infty} \int_{0}^{\tau} d \tau^{\prime} e^{2 \mu_{1}\left(\tau-\tau^{\prime}\right)} \mathbf{q}_{1}^{\dagger}\left(\tau^{\prime}\right) \mathcal{N}\left(\tau^{\prime}\right) \mathbf{q}_{1}^{*}\left(\tau^{\prime}\right),
$$

is a $T$-periodic function.

Let us now prove the expressions above. First, we introduce the quadrature vector $\mathbf{R}=\mathcal{U}\left(\beta, \beta^{+}\right)^{T} / \sqrt{\gamma}$. Within the positive $P$ representation the elements of the long-time mean vector $\overline{\mathbf{d}}$ and covariance matrix $\bar{V}$ are found as $\bar{d}_{m}(\tau)=\lim _{\tau \rightarrow \infty}\left\langle R_{m}(\tau)\right\rangle$ and $\bar{V}_{m n}(\tau)=\delta_{m n}+$ $\lim _{\tau \rightarrow \infty}\left\langle\delta R_{m}(\tau) \delta R_{n}(\tau)\right\rangle$, where $\delta R_{m}=R_{m}-\left\langle R_{m}\right\rangle$ [56]. Next, note that the condition $\mathbf{q}_{0}^{\dagger}(\tau) \mathbf{b}(\tau)=0$ allows us to write the quantum fluctuations as $\mathbf{b}(\tau)=c_{1}(\tau) \mathbf{p}_{1}(\tau)$, where we define the projection $c_{1}(\tau)=\mathbf{q}_{1}^{\dagger}(\tau) \mathbf{b}(\tau)$. Using the expansion (3), we can then write the quadrature vector as $\sqrt{\gamma} \mathbf{R}(\tau)=\mathcal{U}\left[\bar{\beta}(\tau), \bar{\beta}^{+}(\tau)\right]^{T}+c_{1}(\tau) \mathcal{U} \mathbf{p}_{1}(\tau)$, whose stochastic properties are all then concentrated on $c_{1}(\tau)$. On the other hand, applying $\mathbf{q}_{1}^{\dagger}(\tau)$ on Eq. (4) we find $\dot{c}_{1}=\mu_{1} c_{1}+$ $\sqrt{\gamma} \mathbf{q}_{1}^{\dagger}(\tau) \mathbf{n}(\tau)$, whose solution leads to the moments $\lim _{\tau \rightarrow \infty}\left\langle c_{1}(\tau)\right\rangle=0$ and $\lim _{\tau \rightarrow \infty}\left\langle c_{1}^{2}(\tau)\right\rangle=\gamma C(\tau)$, which provide the mean vector and covariance matrix in Eq. (10).

The steady state associated with the expansion (3) of the stochastic variables is then given by the balanced mixture

$$
\bar{W}(\mathbf{r})=\int_{0}^{T} \frac{d \theta}{T} W(\mathbf{r} ; \tau+\theta)=\int_{0}^{T} \frac{d \theta}{T} W(\mathbf{r} ; \theta) .
$$

In Fig. 2 we compare the Wigner function (12) with the one obtained by exact simulation [57] of the master equation (1). We find very good agreement even for relatively large $\gamma$, where quantum fluctuations are still quite relevant, as can be appreciated.
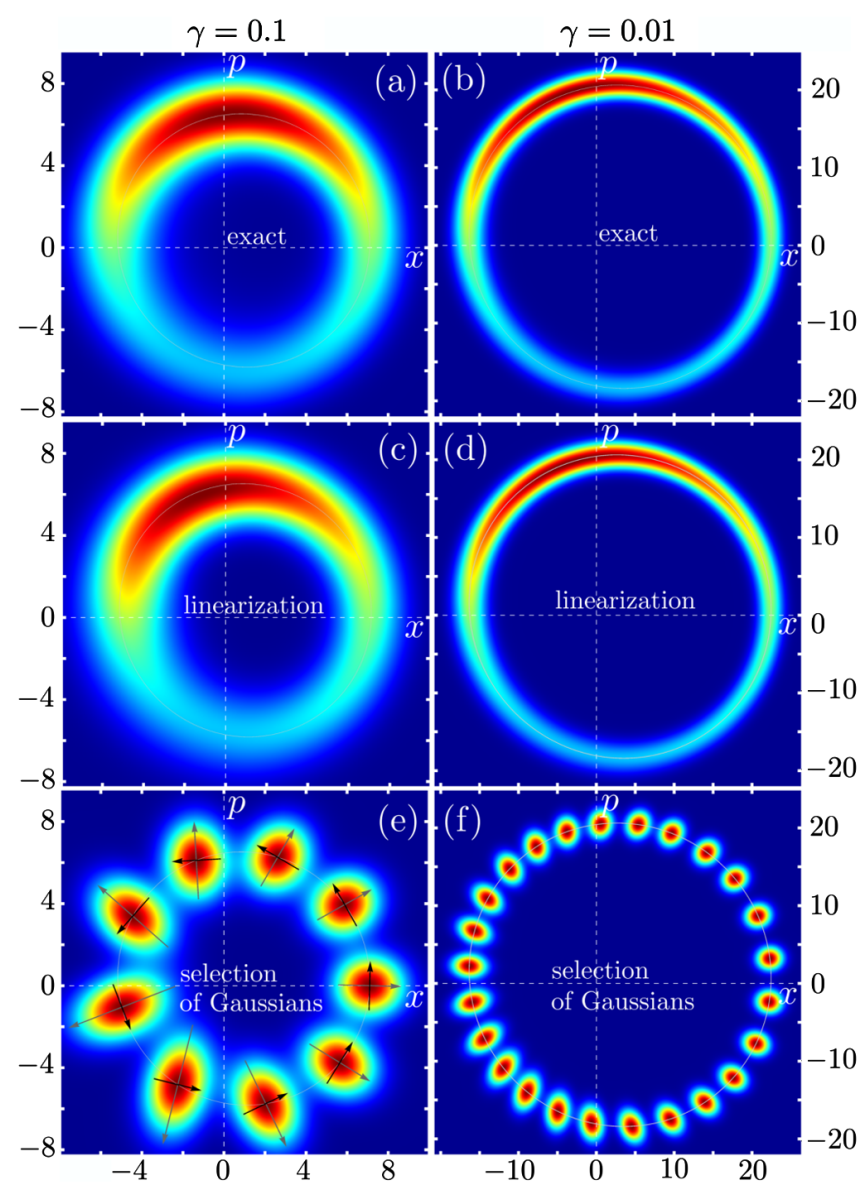

FIG. 2. Steady-state Wigner functions of the driven VdP oscillator, Eq. (1), for $\Delta=\sqrt{0.4}, F=\sqrt{0.1}$, and two values of $\gamma, 0.1$ and 0.01 . In (a) and (b) we show the exact solutions, to be compared with the mixture of Gaussians (c),(d) obtained through linearization, see Eq. (12). In (e) and (f) we show a few of these Gaussian states at selected points of the trajectory, in order to see how these change along the limit cycle. Note how, as shown explicitly in (e), all the Gaussians carry vacuum fluctuations along the direction defined by the $\mathbf{q}_{0}(\tau)$ Floquet eigenvector (black arrows), with varying fluctuations along the $\mathbf{p}_{1}(\tau)$ direction (gray arrows).

This Wigner function has a very suggestive interpretation, see Fig. 2. First, Eq. (10a) tells us that the Gaussian states are centered along the points of the limit cycle's trajectory, as expected. As for quantum fluctuations, note that the eigenvalues of the covariance matrix $\bar{V}(\theta)$ are 1 and $\operatorname{det}\{\bar{V}(\theta)\}$, which inform us about the variance along the principal axes of the uncertainty ellipse. It is easy to check that the directions of these principal axes follow the vectors $\mathcal{U} \mathbf{q}_{0}(\theta)$ and $\mathcal{U} \mathbf{p}_{1}(\theta)$ for the 1 and $\operatorname{det}\{\bar{V}(\theta)\}$ eigenvalues, respectively (see Fig. 2). Hence, the quadrature of the Gaussian state that goes in the direction of $\mathbf{q}_{0}(\theta)$ (Goldstone mode) carries vacuum fluctuations, which one can trace back to the condition $\mathbf{q}_{0}^{\dagger}(\theta) \mathbf{b}(\theta)=0$ that the method naturally demands. On the other hand, since in principle all physical covariance matrices satisfy $\operatorname{det}\{\bar{V}\} \geq 1$ 
(uncertainty principle) [56], this seems to suggest that the quadrature going in the direction of $\mathbf{p}_{1}(\theta)$ carries fluctuations above the shot noise limit. While this is indeed the case for the VdP oscillator studied here, our experience with other nonlinear systems [14] tells us that we could find $\operatorname{det}\{\bar{V}(\theta)\}<1$ (squeezing below shot noise) without violating the uncertainty principle. This is because the two quadratures of each Gaussian state are not conjugate variables, but they are both conjugate to the diffusing variable $\theta$ [14], which is completely undetermined in the steady state.

Conclusions.-In this Letter we have introduced a linearization method capable of dealing with quantum nonlinear systems in the regime where they show spontaneous limit-cycle formation. The technique keeps the simplicity of standard linearization around stationary solutions. It requires finding the fundamental matrix of the Floquet method over a period of the cycle by solving a linear initial value problem with time-periodic coefficients. Only two equations are added with each mode that is introduced in the problem, giving the method a linear scaling with the size of the system that makes it suitable for complex driven-dissipative many-body problems such as optomechanical arrays [45-49]. Moreover, the linearity of the equations should give efficient access also to dynamical objects such as multitime correlation functions, which are sensitive to the diffusion of $\theta$ in general, and are of crucial relevance for experiments $[3,4,6,52]$ and the emergent field of quantum synchronization [40-42,58-63].

We thank Florian Marquardt for important suggestions and comments. Our work also benefited from discussions with Alessandro Farace, Alejandro González-Tudela, and Eugenio Roldán. This work was supported by the European Research Council starting grant OPTOMECH, and by the Ministerio de Economía y Competitividad of the Spanish Government and the Fondo Europeo de Desarrollo Regional through project FIS2014-60715-P.

[1] J. P. Dowling and G. J. Milburn, Phil. Trans. R. Soc. A 361, 1655 (2003).

[2] M. Benito, C. Sánchez Muñoz, and C. Navarrete-Benlloch, Phys. Rev. A 93, 023846 (2016).

[3] C. W. Gardiner and P. Zoller, Quantum Noise (SpringerVerlag, Berlin, Heidelberg, 2004).

[4] C. Gardiner and P. Zoller, The Quantum World of Ultra-Cold Atoms and Light, Book I (Imperial College Press, London, 2014).

[5] H.-P. Breuer and F. Petruccione, The Theory of Open Quantum Systems (Oxford University Press, New York, 2002).

[6] H. J. Carmichael, Statistical Methods in Quantum Optics 1 (Springer-Verlag, Berlin, Heidelberg, 2002).

[7] P. D. Drummond, K. J. McNeil, and D. F. Walls, Opt. Acta 28, 211 (1981).

[8] L. A. Lugiato and G. Strini, Opt. Commun. 41, 67 (1982).
[9] C. Navarrete-Benlloch, E. Roldán, Y. Chang, and T. Shi, Opt. Express 22, 24010 (2014).

[10] I. Pérez-Arjona, E. Roldán, and G. J. de Valcárcel, Europhys. Lett. 74, 247 (2006).

[11] I. Pérez-Arjona, E. Roldán, and G. J. de Valcárcel, Phys. Rev. A 75, 063802 (2007).

[12] C. Navarrete-Benlloch, E. Roldán, and G. J. de Valcárcel, Phys. Rev. Lett. 100, 203601 (2008).

[13] C. Navarrete-Benlloch, A. Romanelli, E. Roldán, and G. J. de Valcárcel, Phys. Rev. A 81, 043829 (2010).

[14] C. Navarrete-Benlloch, Universitat de València, Ph.D. thesis, 2011; arXiv:1504.05917.

[15] A. S. Lane, M. D. Reid, and D. F. Walls, Phys. Rev. A 38, 788 (1988).

[16] M. D. Reid and P. D. Drummond, Phys. Rev. Lett. 60, 2731 (1988).

[17] M. D. Reid and P. D. Drummond, Phys. Rev. A 40, 4493 (1989).

[18] F. V. Garcia-Ferrer, C. Navarrete-Benlloch, G. J. de Valcárcel, and E. Roldán, IEEE J. Quantum Electron. 45, 1404 (2009).

[19] F. V. Garcia-Ferrer, C. Navarrete-Benlloch, G. J. de Valcárcel, and E. Roldán, Opt. Lett. 35, 2194 (2010).

[20] P. Bergé, Y. Pomeau, and C. Vidal, Order within Chaos (Hermann, Paris, 1984).

[21] S. H. Strogatz, Nonlinear Dynamics and Chaos (Perseus Books, Reading, 1994).

[22] R. Grimshaw, Nonlinear Ordinary Differential Equations (Blackwell Scientific, London, 1990).

[23] G. Iooss and D. D. Joseph, Elementary Stability and Bifurcation Theory (Springer, Berlin, 1990).

[24] E. H. M. Hogenboom, W. Klische, C. O. Weiss, and A. Godone, Phys. Rev. Lett. 55, 2571 (1985).

[25] C. Y. Wang, L. Diehl, A. Gordon, C. Jirauschek, F. X. Kärtner, A. Belyanin, D. Bour, S. Corzine, G. Höfler, M. Troccoli, J. Faist, and F. Capasso, Phys. Rev. A 75, 031802(R) (2007)

[26] T. Voigt, M. O. Lenz, F. Mitschke, E. Roldán, and G. J. de Valcárcel, Appl. Phys. B 79, 175 (2004).

[27] Y. Yu, W. Xue, E. Semenova, K. Yvind, and J. Mork, Nat. Photonics 11, 81 (2017).

[28] P. D. Drummond, K. J. McNeil, and D. F. Walls, Opt. Acta 27, 321 (1980).

[29] M. Bache, P. Lodahl, A. V. Mamaev, M. Marcus, and M. Saffman, Phys. Rev. A 65, 033811 (2002).

[30] F. Marquardt, J. G. E. Harris, and S. M. Girvin, Phys. Rev. Lett. 96, 103901 (2006).

[31] M. Ludwig, B. Kubala, and F. Marquardt, New J. Phys. 10, 095013 (2008).

[32] T. Carmon, H. Rokhsari, L. Yang, T. J. Kippenberg, and K. J. Vahala, Phys. Rev. Lett. 94, 223902 (2005).

[33] T. J. Kippenberg, H. Rokhsari, T. Carmon, A. Scherer, and K. J. Vahala, Phys. Rev. Lett. 95, 033901 (2005).

[34] H. Rokhsari, T. J. Kippenberg, T. Carmon, and K. J. Vahala, Opt. Express 13, 5293 (2005).

[35] A. G. Krause, J. T. Hill, M. Ludwig, A. H. Safavi-Naeini, J. Chan, F. Marquardt, and O. Painter, Phys. Rev. Lett. 115, 233601 (2015).

[36] F. M. Buters, H. J. Eerkens, K. Heeck, M. J. Weaver, B. Pepper, S. de Man, and D. Bouwmeester, Phys. Rev. A 92, 013811 (2015) 
[37] M. Aspelmeyer, T. J. Kippenberg, and F. Marquardt, Rev. Mod. Phys. 86, 1391 (2014).

[38] H. Nakatsuka, S. Asaka, H. Itoh, K. Ikeda, and M. Matsuoka, Phys. Rev. Lett. 50, 109 (1983).

[39] J.-J. Zondy, D. Kolker, and F. N. C. Wong, Phys. Rev. Lett. 93, 043902 (2004).

[40] S. Walter, A. Nunnenkamp, and C. Bruder, Phys. Rev. Lett. 112, 094102 (2014).

[41] T. Weiss, S. Walter, and F. Marquardt, Phys. Rev. A 95, 041802(R) (2017).

[42] N. Lörch, E. Amitai, A. Nunnenkamp, and C. Bruder, Phys. Rev. Lett. 117, 073601 (2016).

[43] J. Qian, A. A. Clerk, K. Hammerer, and F. Marquardt, Phys. Rev. Lett. 109, 253601 (2012).

[44] N. Lörch, J. Qian, A. Clerk, F. Marquardt, and K. Hammerer, Phys. Rev. X 4, 011015 (2014).

[45] G. Heinrich, M. Ludwig, J. Qian, B. Kubala, and F. Marquardt, Phys. Rev. Lett. 107, 043603 (2011).

[46] M. Ludwig and F. Marquardt, Phys. Rev. Lett. 111, 073603 (2013).

[47] R. Lauter, C. Brendel, S. J. M. Habraken, and F. Marquardt, Phys. Rev. E 92, 012902 (2015).

[48] T. Weiss, A. Kronwald, and F. Marquardt, New J. Phys. 18, 013043 (2016).

[49] R. Lauter, A. Mitra, and F. Marquardt, Phys. Rev. E 96, 012220 (2017).

[50] W. P. Schleich, Quantum Optics in Phase Space (WileyVCH, Berlin, 2001).

[51] P. D. Drummond and C. W. Gardiner, J. Phys. A 13, 2353 (1980).
[52] H. J. Carmichael, Statistical Methods in Quantum Optics 2 (Springer-Verlag, Berlin, 2008).

[53] C. W. Gardiner, Stochastic Methods: A Handbook for the Natural and Social Sciences, 4th ed. (Springer-Verlag, Berlin, 2009).

[54] See Supplemental Material at http://link.aps.org/ supplemental/10.1103/PhysRevLett.119.133601, where we derive the stochastic equations of the system, prove the relevant properties of the Floquet eigensystem, and review the phase diagram of the driven $\mathrm{VdP}$ oscillator in the classical limit.

[55] E. A. Coddington and N. Levinson, Theory of Ordinary Differential Equations (McGraw-Hill, New York, 1955).

[56] C. Navarrete-Benlloch, An Introduction to the Formalism of Quantum Information with Continuous Variables (Morgan \& Claypool and IOP, Bristol, 2015).

[57] C. Navarrete-Benlloch, arXiv:1504.05266.

[58] G. L. Giorgi, F. Galve, G. Manzano, P. Colet, and R. Zambrini, Phys. Rev. A 85, 052101 (2012).

[59] G. Manzano, F. Galve, G. L. Giorgi, E. Hernández-García, and R. Zambrini, Sci. Rep. 3, 1439 (2013).

[60] T. E. Lee and H. R. Sadeghpour, Phys. Rev. Lett. 111, 234101 (2013).

[61] A. Mari, A. Farace, N. Didier, V. Giovannetti, and R. Fazio, Phys. Rev. Lett. 111, 103605 (2013).

[62] S. Walter, A. Nunnenkamp, and C. Bruder, Ann. Phys. (Amsterdam) 527, 131 (2014).

[63] C. Benedetti, F. Galve, A. Mandarino, M. G. A. Paris, and R. Zambrini, Phys. Rev. A 94, 052118 (2016). 